TITLE:

\title{
Molecular ion implanter equipped with liquid-metal alloy ion source
}

$\operatorname{AUTHOR}(S):$

Gotoh, Y; Tsuji, H; Ishikawa, J

\section{CITATION:}

Gotoh, Y ...[et al]. Molecular ion implanter equipped with liquid-metal alloy ion source. REVIEW OF SCIENTIFIC INSTRUMENTS 2000, 71(2): 780782

\section{ISSUE DATE:}

2000-02

URL:

http://hdl.handle.net/2433/39798

\section{RIGHT:}

Copyright 2000 American Institute of Physics. This article may be downloaded for personal use only. Any other use requires prior permission of the author and the American Institute of Physics. 


\title{
Molecular ion implanter equipped with liquid-metal alloy ion source
}

\author{
Y. Gotoh, ${ }^{\text {a) }} \mathrm{H}$. Tsuji, and J. Ishikawa \\ Department of Electronic Science and Engineering, Kyoto University, Yoshida-honmachi, Sakyo-ku, \\ Kyoto 606-8501, Japan
}

(Presented on 6 September 1999)

\begin{abstract}
Molecular ion implanter was developed with a liquid-metal alloy ion source. Use of a liquid-metal alloy ion source enables us to generate various kinds of molecular ions. To apply liquid-metal ion source to a general ion implanter, it is necessary to converge the divergent beam. We adopted the lens system we have already developed, and examined its performance by computer simulation and experiments. An example of molecular ion implantation was demonstrated with gold-antimony ion source. $24 \mathrm{keV} \mathrm{AuSb}{ }^{2+}$ was implanted into silicon, and presence of gold and antimony atoms was confirmed by Rutherford backscattering spectrometry and particle induced x-ray emission measurements. () 2000 American Institute of Physics. [S0034-6748(00)58902-3]
\end{abstract}

\section{INTRODUCTION}

Liquid-metal ion sources (LMISs) have been utilized almost only for focused ion beam techology, ${ }^{1}$ but they have many attractive features as a general metal ion source: simple structure, low power consumption, and no gas flow which is compatible to ultrahigh vacuum systems. Thus, various kinds of application is expected including materials science. A LMIS produces not only singly charged monomer ions, but also doubly charged monomer ions, as well as doubly charged molecular ions. We have developed goldantimony $(\mathrm{Au}-\mathrm{Sb})$ ion sources ${ }^{2}$ and found that intense molecular ions of $\mathrm{Au}_{m} \mathrm{Sb}_{n}^{k+}$ were produced. Especially, the intensity of $\mathrm{AuSb}^{2+}$ reached about $10 \%$ of total ion current and almost half of the most dominant ion species, $\mathrm{Au}^{+}$. The fraction of the molecular intensity did not change with an increase in the emission current. ${ }^{3}$ Other alloy ion sources also yield intense molecular ions. ${ }^{4}$ Production of molecular ions is one of the unique feature of the ion source whose ionization mechanism is based on the surface effect.

On the other hand, molecular implantation was performed with homogeneous molecules such as $\mathrm{N}_{2}$ and $\mathrm{O}_{2}$, or hydrides of boron, phosphine and arsenic. ${ }^{5}$ Also decaborane ${ }^{6}$ implantation attracted much interest for formation of a shallower junction. We have proposed molecular ion implantation which emphasizes the introduction of heterogeneous atoms into solids. ${ }^{7}$ This molecular ion implantation has two advantages: introduction of different elements to similar depth at a single implantation, and simultaneous implantation at the same position. However, we did not demonstrate any typical example because production of heterogeneous molecular ion is generally difficult. However, as stated above, using LMIS, it becomes possible. Combination of $\mathrm{Au}$ and $\mathrm{Sb}$ might make a metallized layer and $n$-type semiconductor junction with very thin region. The present article deals with the performance of a molecular ion implanter equipped with a LM alloy ion source.

${ }^{a)}$ Electronic mail: ygotoh@kuee.kyoto-u.ac.jp

\section{PERFORMANCE OF ION IMPLANTER WITH LIQUID-METAL ION SOURCE}

\section{A. Configuration of ion implanter}

One of the most significant difficulties to apply a LMIS to an ion implanter, is how to converge the divergent beam. We have already developed the ion beam transport system. ${ }^{8,9}$ The present implantation system was a slightly modified version, as shown in Fig. 1. In this system, divergent beam is converged by a source lens, and later again adjusted the focal length to match the mass separator by a postlens. The source lens, which is illustrated as an insert of Fig. 1, consists of three electrodes, which compose physically asymmetric lens. The extracted ion beam is converged by this lens at the early stage of its divergence. The source lens can form almost a parallel beam. However, beamlets close to beam envelop have shorter focal length due to spherical aberration, thus excess focusing is not preferred. Furthermore, focusing just after extraction gives large image magnification, so it is necessary to use another lens system. In the present study, cylindrical einzel lens with the inner diameter of $80 \mathrm{~mm}$ was used as a postlens. In the previous system, einzel lens with the inner diameter of $60 \mathrm{~mm}$ was set just after the source lens. ${ }^{9}$ The mass separator was a sector magnet with the deflection angle of $60^{\circ}$, and the radius of the curvature of 600 $\mathrm{mm}$.

\section{B. Evaluation of optics}

We estimated the optical performance of the implanter. As described above, after passing through the source lens, each beamlet no longer has the same object point due to spherical aberration. The trajectory for each beamlet within the source lens was calculated by trajectory calculation based on the numerical integration of equation of motion. The potential distribution within the lens were calculated by finite difference method. The entrance aperture of the present source lens allows the beam upto the divergent half angle of $25^{\circ}$, thus 15 beamlets including on axis beam was analyzed. In order to simplify the analysis, radially emitted beam was assumed at the entrance of the source lens. The potentials of 


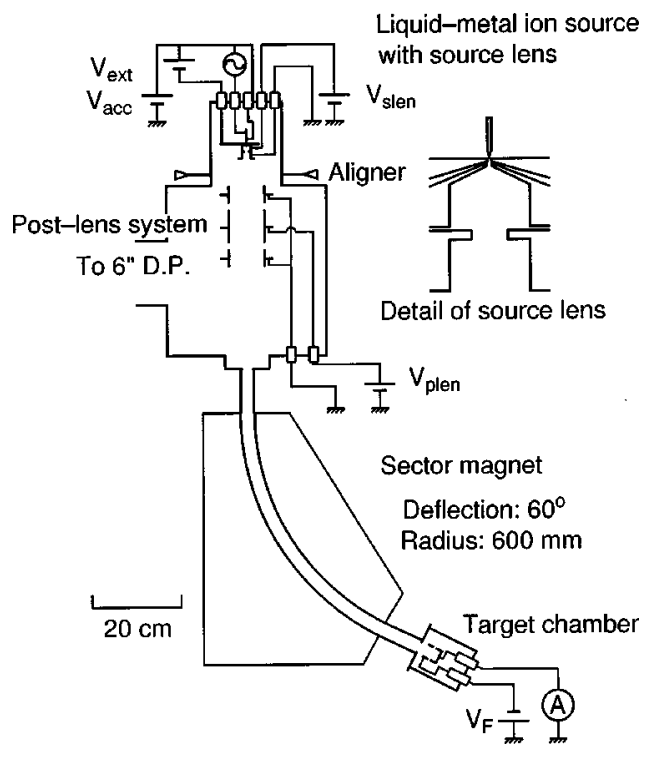

FIG. 1. Schematic diagram of ion implanter.

ion emitter, extraction electrode, center lens electrode, and last electrode were $20 \mathrm{kV}, 12 \mathrm{kV}, 18 \mathrm{kV}$, and $0 \mathrm{~V}$. The outer 5 beamlets hit the lens electrode and 10 beamlets passed the source lens. We assumed the ion beam includes energy spread of $\pm 100 \mathrm{eV}$.

From the above calculation, we obtain the radial position and the component of radial velocity of each beamlet. Since the beam diameter is less than $10 \mathrm{~mm}$, we can treat the following optical elements with the first order approximation. The focal point of each beamlet was calculated using a given characteristics published as a table. ${ }^{10}$ Transport matrix of sector magnet was also given in the literature. ${ }^{11}$

Figures 2(a)-2(d) show the phase space diagram for the ion beam at the various point of the implanter. Figures $2(a)-$

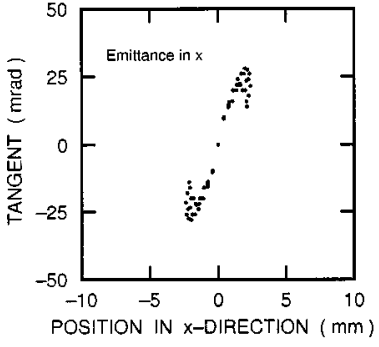

(a)

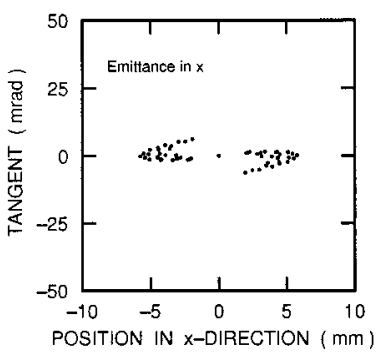

(c)

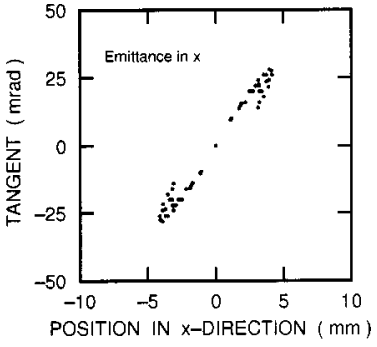

(b)

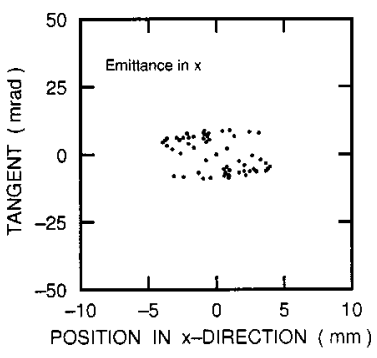

(d)
FIG. 2. Phase space diagram of the ion beam at various points of the implanter. (a) At the exit of the source lens, (b) at the entrance of postlens, (c) at the entrance of mass separator, and (d) at the exit of mass separator.
TABLE I. Condition of molecular ion implantation.

\begin{tabular}{ll}
\hline \hline \multicolumn{1}{c}{ Terms } & \multicolumn{1}{c}{ Conditions } \\
\hline Ion species & $\mathrm{AuSb}^{2+}$ \\
Ion energy & $24 \mathrm{keV}$ \\
Ion current & $0.6 \mu \mathrm{A}$, equivalent to $0.2 \mu \mathrm{A} \mathrm{cm}^{-2}$ \\
Ion dose & $6.7 \times 10^{15}$ ions $\mathrm{cm}^{-2}$ \\
Substrate & $n-\mathrm{Si}(100)$ \\
Ion incident angle & $7^{\circ}$ off with respect to substrate normal \\
\hline \hline
\end{tabular}

2(d) are those at the exit of the source lens, just in front of the postlens, just in front of the mass separator, and at the exit of the mass separator. The initial beam is slightly diverging, but after passing through the postlens, the beam is formed to be almost parallel. The 10 beamlets which passed through the source lens could be transported to the target. Based on the radial current distribution of In ion source at the source current of $200 \mu \mathrm{A}$, the current is almost $38 \mu \mathrm{A}$. We have obtained the current of approximately $40 \mu \mathrm{A}$ with $\mathrm{Ga}$ ion source, which agrees well to the present result.

\section{Separation of molecules}

The ion species which appear close to the $\mathrm{AuSb}^{2+}($ mass/charge $=160)$ was $\mathrm{Sb}^{+}($mass/charge $=122)$, and $\mathrm{Sb}_{3}^{2+}($ mass/charge $=183) . \Delta m / m$ 's are 0.24 and 0.14 , which can be well separated by the present mass separator. If $\mathrm{AuSb}^{+}$is used, $\mathrm{Au}_{3}^{2+}$ (mass/charge $\left.=295.5\right)$ and $\mathrm{AuSb}_{4}^{2+}$ $($ mass/charge $=342.5)$ appear close to $\mathrm{AuSb}^{+}$(mass/charge $=319$ ) thus separation of these ions is difficult.

\section{MOLECULAR IMPLANTATION}

\section{A. Sample preparation}

Molecular ion implantation was performed with the $\mathrm{AuSb}_{2}$ ion source. ${ }^{2}$ As described above, use of $\mathrm{AuSb}^{2+}$ is preferred to avoid cross contamination of different molecules. We implanted with the condition written in Table I. The ion current during implantaion was $0.6 \mu \mathrm{A}$. Taking the fraction of $\mathrm{AuSb}^{2+}$ ions in the total beam current (7.4\%), transported current was $8.1 \mu \mathrm{A}$, if we assume the ion beam consists of a single ion species. This value is lower than the above calculated values, and it would be attributed both to low angular intensity growth for $\mathrm{Au}-\mathrm{Sb}$ ion source ${ }^{3}$ and to lower acceleration voltage of $12 \mathrm{kV}$.

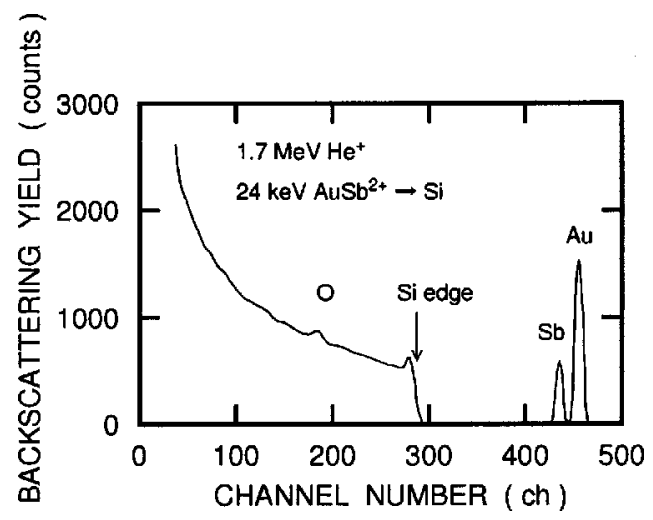

FIG. 3. RBS spectrum of the molecular ion implanted sample. 


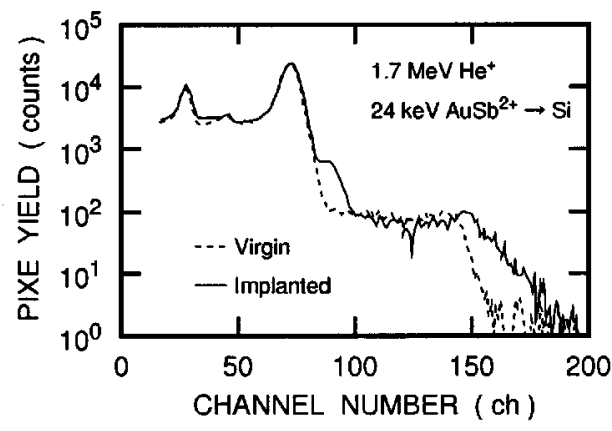

(a)

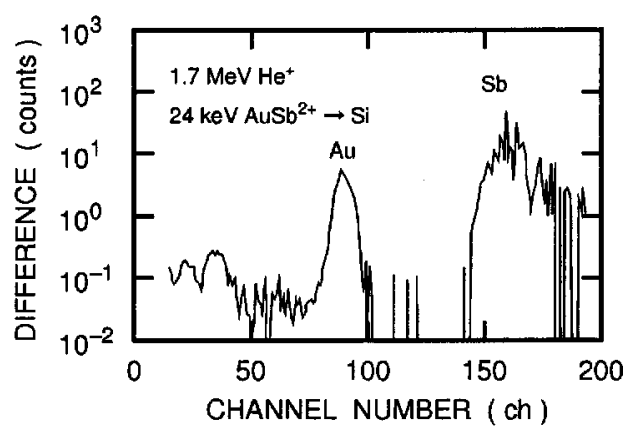

(b)

FIG. 4. PIXE spectrum of molecular ion implanted sample. (a) Signals from implanted sample and virgin substrate and (b) difference of two signals normalized by virgin signal.

\section{B. Analysis of implanted sample}

In order to confirm the presence of the implanted atoms, characterization of implanted sample was performed. Figures 3 and 4 indicate the spectra of Rutherford backscattering spectrometry (RBS) and particle induced x-ray emission (PIXE) measurements, respectively. The probe beam was 1.7 $\mathrm{MeV} \mathrm{He}^{+}$and scattering angle was $135^{\circ}$. From the RBS spectrum, two peaks due to heavy atoms can be seen. The positions of these peaks well agree with those for $\mathrm{Au}$ and $\mathrm{Sb}$. Peak area ratio of $\mathrm{Sb} / \mathrm{Au}$ was 0.40 which showed good agreement with the theoretical value of $0.42 .{ }^{12}$ The absolute implanted dose could not be confirmed because the RBS spectrum shows chanelling effect of the substrate silicon, but considered to be within an error of 50\%. Further confirma- tion was performed by PIXE. Figure 4(a) shows the raw data obtained from the implanted and virgin samples. Only a slight difference could be seen, and the difference was enhanced by subtracting the virgin signal from the implanted signal. The difference was normalized by the virgin singal. This result is shown in Fig. 4(b). The peak at the lower energy corresponds to $\mathrm{Au} M \alpha 2.12 \mathrm{keV}$, and that at the higher energy corresponds to $\mathrm{Sb} L \alpha 3.60 \mathrm{keV} .{ }^{13}$ From these measuremnts, simultaneous implantation of both $\mathrm{Au}$ and $\mathrm{Sb}$ was confirmed.

\section{SUMMARY}

Molecular ion implanter equipped with a liquid-metal alloy ion source is proposed and a fundamental result on the molecular ion implantation was demonstrated. $\mathrm{AuSb}^{2+}$ was implanted into a silicon substrate, and the presence of $\mathrm{Au}$ and $\mathrm{Sb}$ atoms were confirmed by RBS and PIXE.

\section{ACKNOWLEDGMENT}

The RBS and PIXE measurements were performed at Quantum Science and Engineering Center, Kyoto University.

${ }^{1}$ J. Orloff, Rev. Sci. Instrum. 64, 1105 (1993).

${ }^{2}$ Y. Gotoh, H. Tsuji, and J. Ishikawa, Jpn. J. Appl. Phys., Part 1 35, 3670 (1996).

${ }^{3}$ Y. Gotoh, H. Tsuji, and J. Ishikawa, Ultramicroscopy 73, 83 (1998).

${ }^{4}$ W. M. Clark, Jr., R. L. Seliger, M. W. Utlaut, A. E. Bell, L. W. Swanson, G. A. Schwind, and J. B. Jergenson, J. Vac. Sci. Technol. B 5, 197 (1987).

${ }^{5}$ A. Yoshida, M. Kitagawa, and T. Hirao, Jpn. J. Appl. Phys., Part 1 32, 2151 (1993).

${ }^{6}$ D. Takeuchi, N. Shimada, J. Matsuo, and I. Yamada, Nucl. Instrum. Methods Phys. Res. B 121, 345 (1997).

${ }^{7}$ J. Ishikawa, H. Tsuji, M. Mimura, and Y. Gotoh, IEEE Proceedings of the 11th International Conference on Ion Implantation Technology, Austin, 1996 (unpublished), p. 776.

${ }^{8}$ J. Ishikawa, Y. Gotoh, H. Tsuji, and T. Takagi, Nucl. Instrum. Methods Phys. Res. B 21, 186 (1987).

${ }^{9}$ J. Ishikawa, H. Tsuji, T. Kashiwagi, and T. Takagi, Nucl. Instrum. Methods Phys. Res. B 37/38, 155 (1989).

${ }^{10}$ A. B. El-Kareh and J. C. J. El-Kareh, Electron Beams, Lenses, and Optics (Academic, Orlando, FL, 1970).

${ }^{11}$ R. G. Wilson and G. R. Brewer, Ion Beams, With Application To Ion Implantation (Krieger, Malabar, 1979).

${ }^{12}$ W.-K. Chu, J. W. Mayer, and M.-A. Nicolet, Backscattering Spectrometry (Academic, Orlando, FL, 1978).

${ }^{13}$ I. V. Mitchell and J. F. Ziegler, Ion Induced X-Rays, edited by J. W. Mayer and E. Rimini, Ion Beam Handbook of Material Analysis (Academic, New York, 1977), Chap. 5, p. 311. 